\title{
Engaging Digital Engineering Students in Design Thinking
}

\author{
Hanadi Traifeh ${ }^{1}$, Claudia Nicolai ${ }^{1}$, Reem Refaie ${ }^{1}$, Christoph $_{\text {Meinel }}{ }^{1}$ \\ ${ }^{1}$ Hasso Plattner Institute \\ hanadi.traifeh@hpi.de \\ claudia.nicolai@hpi.de \\ Reem.Abourefaie@guest.hpi.de \\ christoph.meinel@hpi.de
}

\begin{abstract}
This research paper examines the impact of the Design Thinking process in cultivating creative confidence among Digital Engineering students and encouraging them to become more empathetic towards others. The setting for the research is a one-week educational workshop at the HPI School of Design Thinking (D-School) in Potsdam, Germany, called the Global Design Thinking Week (GDTW). The workshop takes place twice a year. This research draws on findings from two GDTW workshops, the first was conducted in September 2017 and the second took place in April 2018. At these workshops, Digital Engineering Students from the Hasso Plattner Institute (HPI) were grouped with other participants who came from Germany and other different countries, and had different educational and cultural backgrounds. The workshops were delivered through theoretical inputs and intensive practical sessions on interviewing, ideation, prototyping and testing. Our hypothesis is: By engaging Digital Engineering students in multidisciplinary teams and introducing them to the Design Thinking methodology, students will have increased confidence in their creative thinking skills. The process also encourages them to adapt the Design Thinking mindset so they may become able to defer judgment, be more open minded, and learn to better empathize with others. Qualitative and quantitative methods such as observation, pre and post workshop survey were used to assess if the Design Thinking process affected the participants' creative confidence and how the students engaged with other team members (especially with those who come from different cultures). The research results show that most participants had doubts about their creative abilities before starting the workshops. They were also not sure about how they could connect with those who do not work in IT or do not understand their culture. These beliefs however changed after day 1 of the workshop and a shift in the learning mood was observed. The research found that the intensive Design Thinking workshop had an immediate and positive impact not only on Digital Engineering students but on all participants' creative confidence, and their willingness to develop better understanding of other cultures and experiences through learning to listen and empathize.
\end{abstract}

Keywords: Creative Confidence, Design Thinking, Empathy, Digital Engineering, Problemsolving. 


\section{Introduction}

Creativity and innovation are highly sought-after capabilities and so is the search for the optimal way to foster them. Experiential teaching of Design Thinking has garnered a good reputation for being a gateway to creativity and innovation. Dunne and Martin (2006) define Design Thinking as "the way designers think: the mental processes they use to design objects, services or systems, as distinct from the end results of elegant and useful products". This is reflected in a global trend of adopting such modalities at universities, corporations and governments, in different fields including education, IT, healthcare and others (Traifeh, Staubitz \& Meinel 2019).

Several frameworks of the Design Thinking processes have been developed by different universities and companies around the world. One of the most popular frameworks is that of the Hasso Plattner Institute of Design at Stanford (known as d.school). The d.school was established in 2004 at Stanford University in the USA. In 2007, a sister school called the HPI School of Design Thinking (known as D-School) was established in Potsdam, Germany, where our study took place. It is worth mentioning that the framework currently used at the D-School of Potsdam is slightly different to that of the d.school of Stanford (Figure 1). However, what unites both frameworks, as well as other popular frameworks, is that all start by understanding the problem at hand, empathizing with the users to identify their needs and then moving to the solution space during which brainstorming, prototyping and testing potential solution ideas take place. Also, they all encourage adapting the Design Thinking mindset and principles such as 'deferring judgment', 'building on each other's idea', 'bias towards action', 'radical collaboration', 'learning from failure' and 'embracing experimentation'.
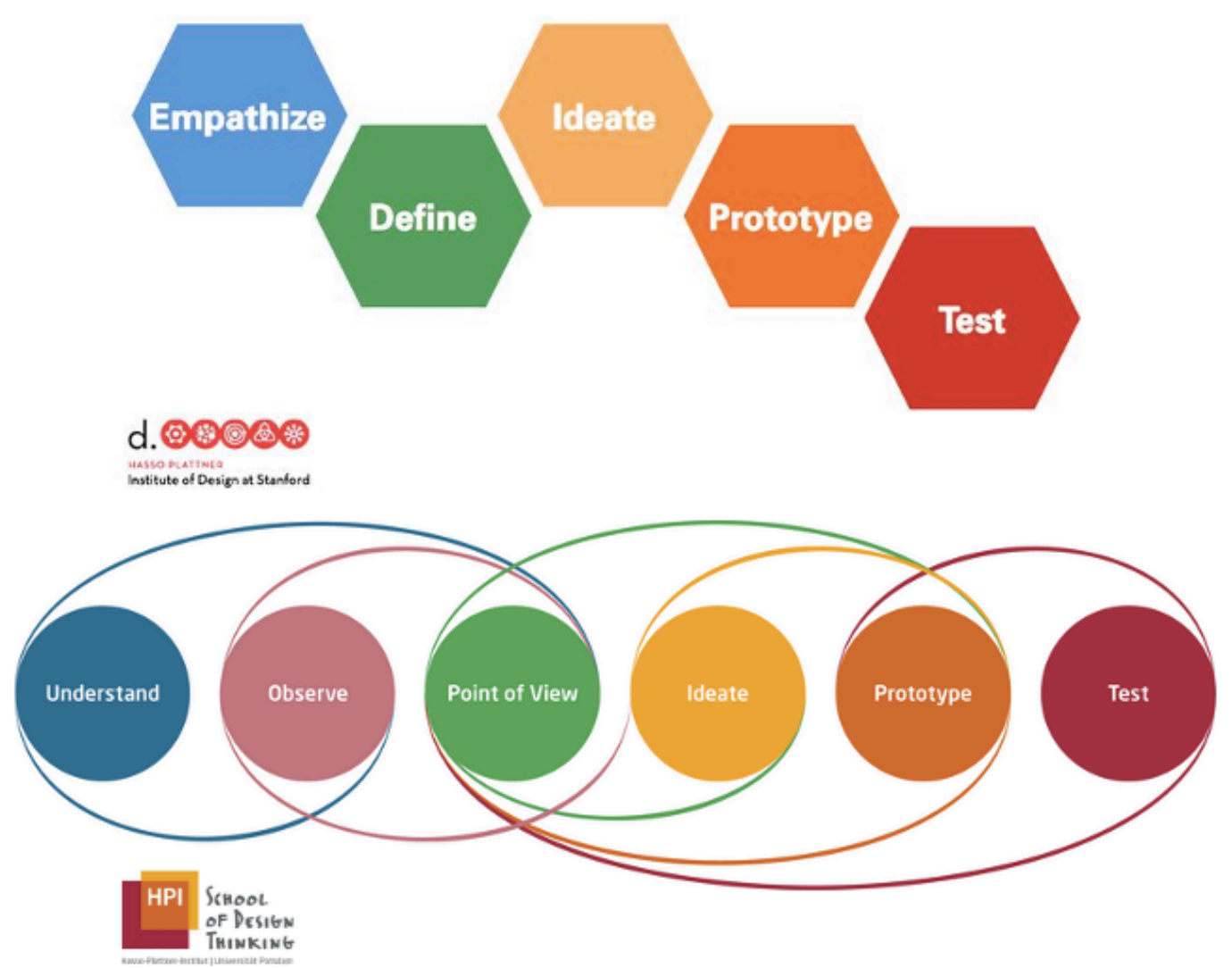
During the Global Design Thinking Week (GDTW), participants go through the Design Thinking process by working on a real-life challenge which is provided by a private/public company, a startup, a for profit/non for-profit organization or an educational institution. Theoretical inputs and intensive practical sessions on interviewing, ideation, prototyping and testing are introduced over one week following the framework. Participants are coached in teams by Design Thinking experts ${ }^{1}$ and expected to fully engage in the process and work with their teammates to find ideas that may contribute to the solution of the challenge at hand. However, there has not been much research done on the impact of Design Thinking (Royalty et. al, 2014). This paper responds to this research dilemma by focusing on the assessment of the effect of the experiential teaching of Design Thinking on fostering students' creative agency and fostering empathy among participants.

Hence, the purpose of this study is to gauge insights on the following two main aspects:

1. Analyze the impact of the Design Thinking process in cultivating creative confidence among participants in general; and Digital Engineering students in particular.

2. Assess the extent to which the Design Thinking process affected the participants' understanding of empathy, their willingness to engage with other team members (who come from different cultures, professional backgrounds or age groups).

\section{Research Study Description}

This study examines the impact of the Design Thinking process in cultivating creative confidence among the Global Design Thinking Week (GDTW) participants in general; Digital Engineering students in particular. It also investigates to what extent the process of Design Thinking can encourage the participants to become more empathetic towards others. While Design Thinking has many definitions (Buchanan 1992), (Cross 2007), the d.school and the DSchool courses are based on a common pedagogy that focuses on an overall process of the Design Thinking methodology. Design Thinking as it is taught at both schools goes beyond explicit pedagogy. The main objective of the pedagogy is to develop future innovators, who, in addition to performing discrete observable skills, also have a characteristic set of attitudes and dispositions that prepares them for exercising creative activity and innovation. According to the Design Thinking teaching model, mindsets and an overall sense of creative confidence are built on top of repeated practice and success with discrete techniques. These mindsets include a 'preference towards action', 'radical collaboration', and 'being human-centered'. Other dispositions, not specified in the model but commonly promoted by Design Thinking experts at both schools, include constant reframing and rapid iteration of concepts and prototypes.

For the purpose of this study, the research was conducted in a one-week educational workshop (GDTW) at the HPI School of Design Thinking (D-School) in Potsdam, Germany in 2017 and the research was repeated the following year, 2018. The GDTW program-which takes place twice a year- aims at providing the participants with a thorough introduction to the iterative

\footnotetext{
${ }^{1}$ Each team consists of five or seven participants and coached by two design thinking coaches.
} 
Design Thinking process. In doing so, the program translates the process into a series of actionbased activities that guide the participants into a reflective journey on a specific topic (real-life challenge). This learning approach combines both problem-based teaching modalities as well as experiential learning in a collaborative learning environment. The workshop also encompasses theoretical inputs and intensive practical sessions. Therefore, the GDTW is implemented as a project-based course, where participants collaborate in each workshop with a project partner (NPOs and NGOs, large enterprises, young start-ups, social organizations, or research institutes and universities). The project partner submits a real-life challenge they are tackling at their workplace, and the GDTW participants work on solving this challenge.

At both workshops, Digital Engineering students from the Hasso Plattner Institute (HPI) were grouped with other participants who came from Germany and also from different countries and had different educational and cultural backgrounds. The first workshop included a total of (53) participants divided into (10) teams. In 2018, the workshop included (65) participations, who were organized into (13) teams. Each team has a total of 5-7 participants and were selected based on the different cultural and disciplines they study or work at. The purpose is to have multidisciplinary teams whereas each participant can bring new views and contribute from her field knowledge to the problem the team is working on. The two groups $(2017,2018)$ were studied with the aim of measuring the impact of the GDTW's pedagogical teaching method in fostering creative confidence among participants, and also in encouraging them to become more empathetic towards others. The research puts an extra emphasis on Digital Engineering students, who are the target audience for the Hasso Plattner Institute for IT-Systems Engineering, Data Engineering and Digital Health in Potsdam. Therefore, the influence of Design Thinking on fostering their creative confidence and measuring their willingness to work in multidisciplinary-multi cultural teams is of high relevance to this study.

\subsection{Research Hypothesis}

By engaging Digital Engineering students in multidisciplinary teams and introducing them to the Design Thinking methodology during a one-week workshop, the participants will have increased confidence in their creative thinking skills. The process will also encourage them to adopt the Design Thinking mindset in the future so they may become able to defer judgment, be more open minded, and learn to better empathize with others.

\subsection{Methodology}

\subsubsection{Royalty et al. Competency-Based Creative Agency Scale}

The Royalty et al. (2014) evaluation methodology offers a scale to measure creative agency as a major outcome of learning Design Thinking. The Competency-Based Creative Agency (CBCA) Scale tests for a total of 11 competencies that were generated from the analysis of data collected from a series of surveys and interviews that were administered at the d.school at Stanford University. The survey targeted a sample of 184 d.school alumni, the majority of whom graduated in 2008 or after. The survey questions aimed to probe the potential role that the d.school pedagogy had on alumni career choice and professional outcomes. The questions mainly asked about the impact on students' current and previous occupations and occupational plans and how they applied what they learned at the d.school in their professional lives. The findings of the survey were enriched with a series of semi-structured interviews, which targeted $16 \mathrm{~d}$.school alumni. The interviews probed deeper into the question of how successful alumni 
perceived what they learned at the d.school and how that influenced their career choices and their professional activities after graduating.

Table 1 shows the CBCA, which highlighted a set of competencies comprised of eleven (11) items that pertained to the Design Thinking process and attest to what Royalty et al. referred to as "Creative confidence". Creative confidence is a new term that Tom and David Kelley $(2013)^{2}$ used to describe a theme of feeling comfortable with creative endeavors and a sense of ability and self-efficacy in the creative domain. Those were translated into Likert-scale selfreported questionnaire with corresponding five response categories: "Not At All" (scale point 1), "A little Confident" (scale point 2), "Moderately Confident" (scale point 3), "Very Confident" (scale point 4) and "Completely Confident" (scale point 5).

Table. 1 Competency- Based Creative Agency (CBCA) Scale

\begin{tabular}{|c|c|c|c|c|c|c|}
\hline \# & Creative Competencies & $\begin{array}{c}1 \\
\text { "Not at } \\
\text { all" }\end{array}$ & $\begin{array}{c}2 \\
\text { "A little } \\
\text { Confident" }\end{array}$ & $\begin{array}{c}3 \\
\text { "Moderately } \\
\text { Confident" }\end{array}$ & $\begin{array}{c}4 \\
\text { "Very } \\
\text { Confident" }\end{array}$ & $\begin{array}{c}5 \\
\text { "Completely } \\
\text { Confident" }\end{array}$ \\
\hline 1 & Creative Idea Sourcing & & & & & \\
\hline 2 & $\begin{array}{l}\text { Comfort with } \\
\text { ambiguity }\end{array}$ & & & & & \\
\hline 3 & $\begin{array}{l}\text { Mastery of one's own } \\
\text { creative process }\end{array}$ & & & & & \\
\hline 4 & $\begin{array}{l}\text { Shaping Creative } \\
\text { Environments }\end{array}$ & & & & & \\
\hline 5 & Anti-perfectionism & & & & & \\
\hline 6 & Prototyping & & & & & \\
\hline 7 & $\begin{array}{l}\text { Perseverance after } \\
\text { Failure }\end{array}$ & & & & & \\
\hline 8 & $\begin{array}{l}\text { Creativity Facilitation } \\
\text { in Others }\end{array}$ & & & & & \\
\hline 9 & Openness & & & & & \\
\hline 10 & $\begin{array}{l}\text { Knowledge of Creative } \\
\text { Process }\end{array}$ & & & & & \\
\hline 11 & $\begin{array}{l}\text { Successful Creative } \\
\text { problem-solving }\end{array}$ & & & & & \\
\hline
\end{tabular}

\footnotetext{
${ }^{2}$ More specifically they defined creative confidence as "believing in your ability to create change in the world around you" (T\&D Kelley, 2013, p. 2)
} 

GDTW

Our study employed the pre-post evaluation design, which was administered through a survey, consisting of fifteen (15) questions. The survey adopts the Competency Based Creative Agency Scale (CBCA), developed at d.school by Royalty et al. (2014). Like the Royalty method, the questionnaire/assessment tool applied in this study comprised of a self-reported scale to evaluate the participants' creative self-efficacy and perception of their own creative capacity. The adapted methodology replicated the CBCA's eleven (11) questions that aimed at assessing the participants' level of confidence with regards to a bundle of skills pertaining to creative thinking and problem-solving. For these, the adopted scale followed the CBCA 1 to 5 model. However, for the purpose of this study, the questionnaire included an additional four (4) questions to probe the participant's cultural sensitivity and empathy, which were not included in Royalty's questionnaire. Like the CBCA, the questionnaire at hand also collected demographic data on the participants' age, gender and professional backgrounds. Table 2 summarizes the competencies examined in the study and the corresponding "How confident...?" questions. For the second section of the questionnaire, participants were asked "How comfortable...?" and the study adapted the scale into a 1 to 4 (forced Likert scale) instead of a 1 to 5 scale as the researchers did not want to provide the safe 'neutral' or evasive option that participants may feel answering when comes to culturally-sensitive questions. The scale for the second section of the questionnaire is "Not At All" (scale point 1), "A little comfortable" (scale point 2), "I don't mind" (scale point 3 ) and "I am fully comfortable" (scale point 4).

Table 2 CBCA- Creative Agency Assessment Tool

\begin{tabular}{|ll|}
\hline \multicolumn{1}{|c|}{ Competencies } & \multicolumn{1}{|c|}{ Question } \\
\hline \multicolumn{1}{|c|}{ Creative Thinking, Problem-Solving, Teamwork and Prototyping } \\
"How confident are you that you could...?"
\end{tabular}




\begin{tabular}{|c|c|}
\hline Prototyping & $\begin{array}{l}\text { Try an approach to a problem or task that you know } \\
\text { may not be the final or best solution }\end{array}$ \\
\hline Perseverance after Failure & $\begin{array}{l}\text { Continue to work on a problem or task after } \\
\text { experiencing a significant failure }\end{array}$ \\
\hline Creativity Facilitation in Others & Help others be more creative \\
\hline Openness & $\begin{array}{l}\text { Change the definition or parameters of a goal, task or } \\
\text { problem you are working on }\end{array}$ \\
\hline Knowledge of Creative Process & Explicitly define or describe your creative process \\
\hline Successful Creative problem-solving & $\begin{array}{l}\text { Solve problems in ways that others would consider } \\
\text { creative }\end{array}$ \\
\hline \multicolumn{2}{|c|}{$\begin{array}{c}\text { Cultural Sensitivity and Empathy } \\
\text { How comfortable are you working with people from...? }\end{array}$} \\
\hline Empathy and openness & Other educational backgrounds \\
\hline Empathy and openness & Other professions \\
\hline Empathy and openness & Other age groups \\
\hline Cultural sensitivity & Other cultures \\
\hline
\end{tabular}

\subsubsection{Limitations}

The study's main limitation is derived from the low response rate. In the two workshops (2017, 2018), most of the participants responded adequately to the pre-survey but only a minority of them responded to the post-survey. To overcome this constraint, the research team matched the responses from the pre-post surveys to ensure consistency. Therefore, the following paragraphs report on the matched data, and not all of the received responses. This amounts to a total of 11 respondents from 2017 and 14 respondents from 2018. The findings are also supported by the notes taken during observation of the teams in both workshops.

\section{Research Findings}

The research shows that only a few of the participants felt a severe lack of confidence with regards to any of the examined competencies. This is reflected in the results below, which show very few indications on the "not at all" category. Most Digital Engineering students had doubts about their creative abilities before starting the workshop. They were also not sure about how they could connect with those who do not work in IT or do not understand their culture. These beliefs however changed after day 1 of the workshop and a shift in the learning mood was observed. Moreover, the research found that the intensive Design Thinking workshop had an immediate and positive impact not only on Digital Engineering students but on all participants' creative confidence, and their willingness to develop better understanding of other cultures and experiences through learning to listen and empathize. 
The following sections report on a set of general competencies in the area of creative problemsolving, teamwork, cultural sensitivity and empathy, which have been associated with the DSchool and d.school teaching models and considered inherent to the Design Thinking process.

\subsection{Creative Idea Sourcing}

The D-School teaching method's main purpose is to guide students from all disciplines into thinking as well as working in more creative ways. However, creativity in itself is an elusive term that is hard to identify, let alone measure. Nevertheless, using the CBCA, this study helps to reflect the participants' self-assessment of their creative skills.

The study confirmed the initial research hypothesis by showing that the participants felt more confident about their ability to find creative inspirations that are unusual or not obviously related to a given problem or task. The pre-survey from 2017 points to one participant feeling a little confident, six participants feeling moderately confident and four participants feeling very confident. These results shifted towards stronger feelings of confidence after completing the workshop. A total of eight participants, five of whom are Digital Engineering students; were either moderately confident or very confident, and three participants (one of them is a Digital Engineering student) reported being completely confident. By the end of the workshop, no participants felt a little confident. The survey conducted in 2018 shows similar results, with most of the participants being moderately confident and they built-up confidence after the workshop by indicating either being very confident or completely confident.

\subsection{Comfort with Ambiguity}

When asked about the ability to effectively work on a problem/task that does not have an obvious or known solution, many of the participants in both workshops showed progress between the pre and the post survey. Before experiencing GDTW, the participants lacked the confidence in their ability to effectively work on a problem, but after they engaged with the Design Thinking methodology throughout the GDTW, the participants' level of confidence increased indicating a positive influence. During our observation of the teams, we noticed that the Digital Engineering students struggled when they started the workshop. According to the comments made by 23 of them, they said that they are not familiar with working with undefined tasks. Their attitude however changed while progressing in the workshops.

\subsection{Redefining problems - Openness}

The ability to define and redefine problems and set goals are cross-cutting skills that are indispensable to everyone regardless of their professional, academic or cultural backgrounds. Design Thinking values these skills and works on fostering them through the exposure to the methodology as well as engaging with others in multidisciplinary teams. The study expects a positive correlation between participating in the GDTW and the increase in confidence levels. Figure 2 supports this hypothesis as the participants reported higher levels of confidence after GDTW. The study from 2018 (Figure 3), reported different results as it showed that there was little to no change in confidence levels before and after the workshop. 


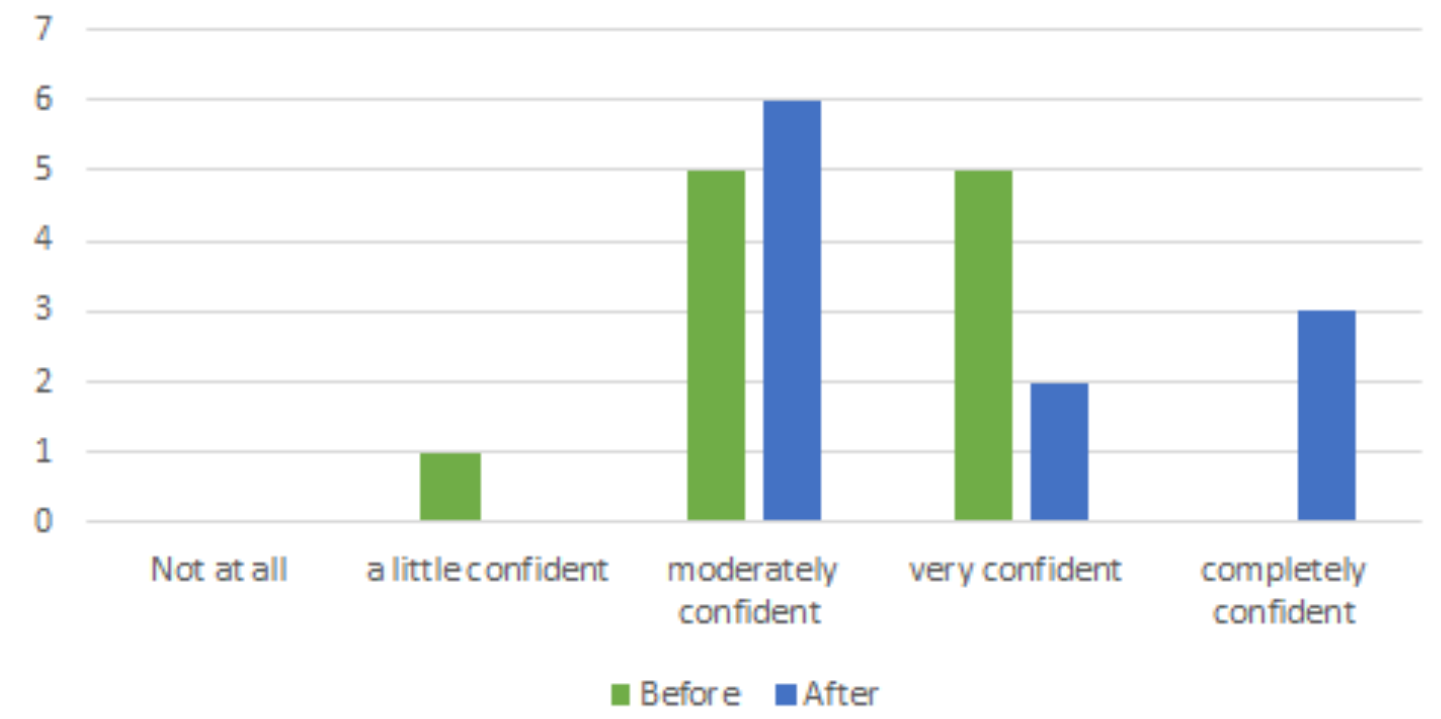

Figure 2. Level of confidence in the ability to define problem and set goal parameters (source: 2017 study)

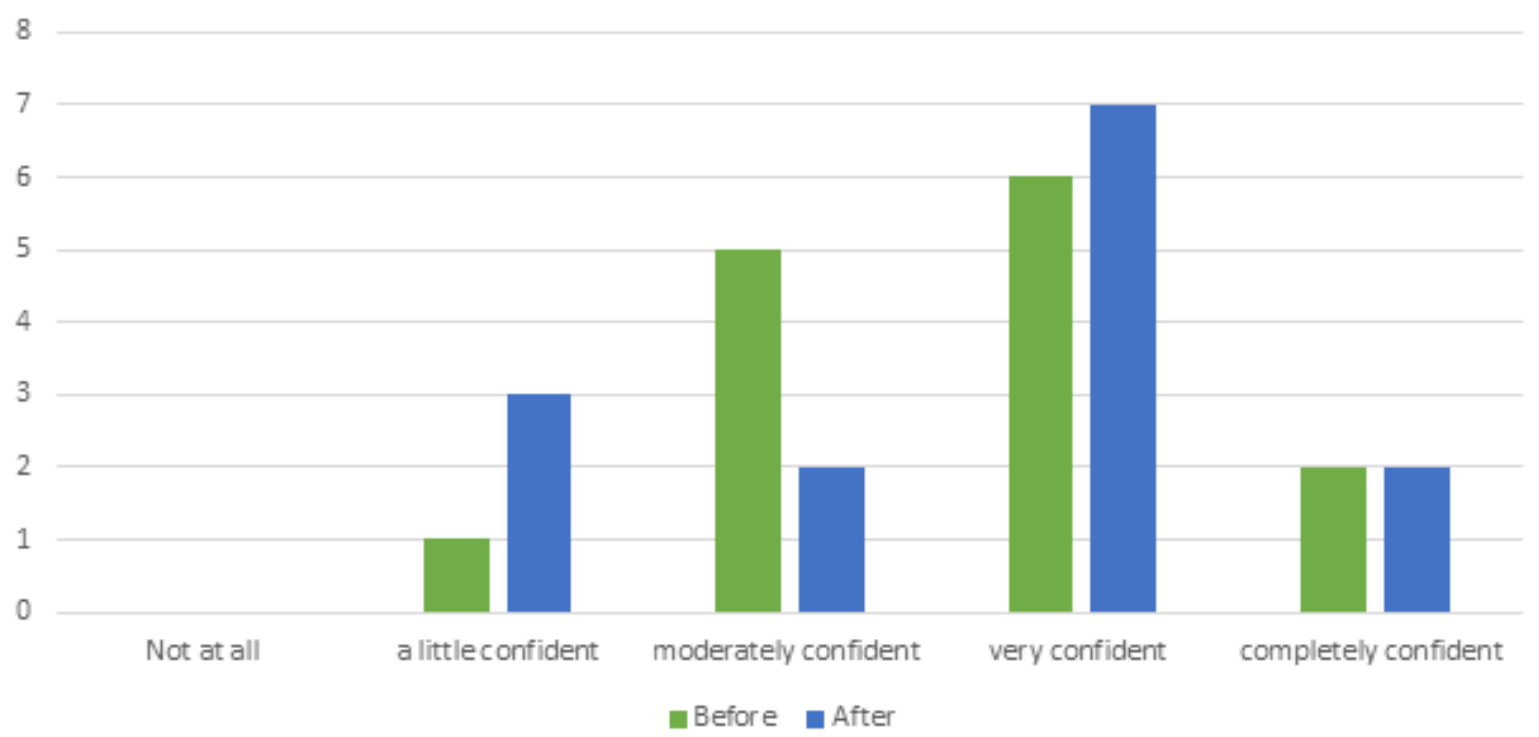

Figure 3. Level of confidence in the ability to define and set goal parameters (source: 2018 study)

\subsection{Building Creative Environments}

The design of spaces to improve creativity, innovation and enhance productivity lies at the heart of Design Thinking. In this respect, the Design Thinking methodology is expected to guide participants to be able to shape their environments to foster creativity. Figure 4 highlights a clear improvement in the participants' confidence after the completion of the GDTW in 2017. Figure 5 shows that the repetition of the study in 2018 mostly affirms those findings with the exception of one participant who moved from being not at all confident before the workshop to being moderately confident after the workshop. 


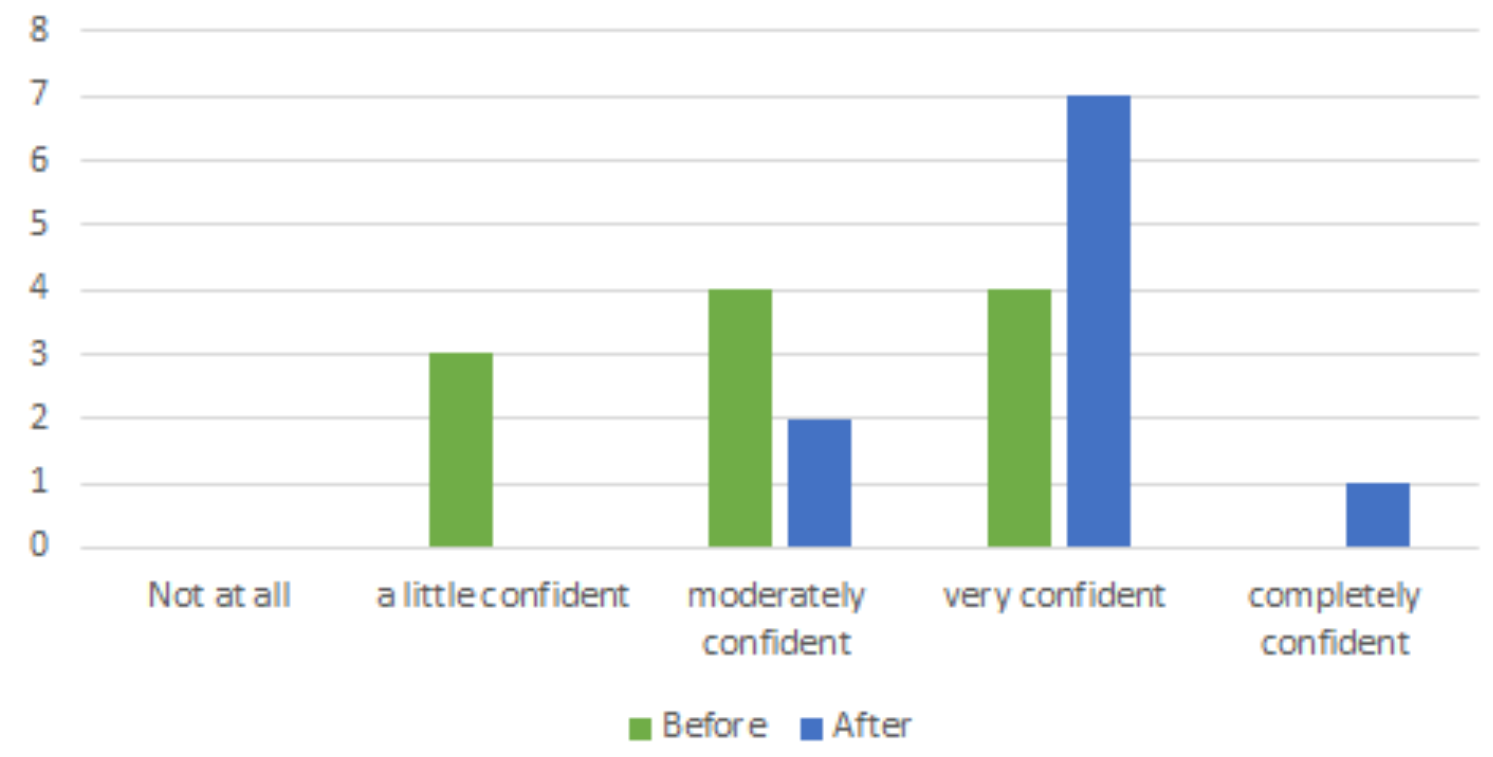

Figure 4. Level of confidence in the ability to shape the external environment to foster creativity (source: 2017 study)

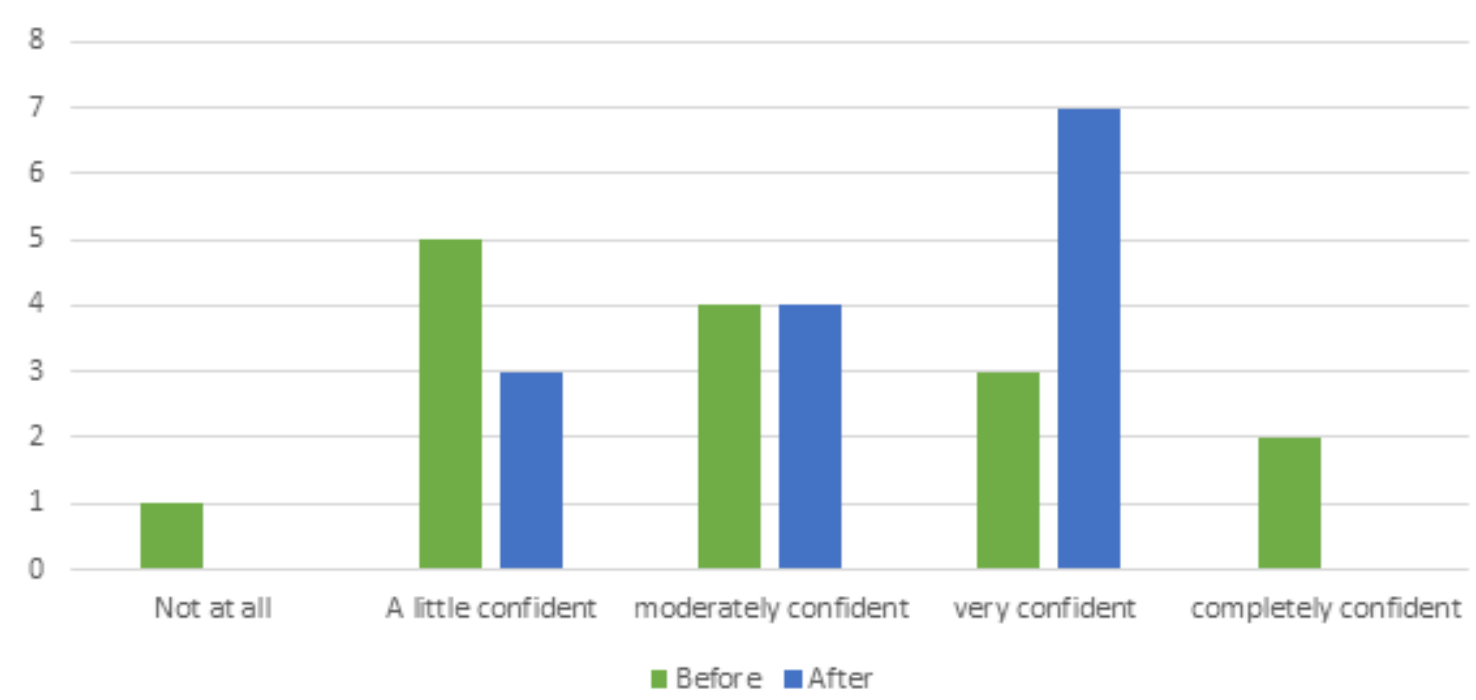

Figure 5. Level of confidence in the ability to shape the external environment to foster creativity (source: 2018 study)

\subsection{Anti-Perfectionism}

Working in multidisciplinary teams is key to applying the Design Thinking methodology. The study aimed to test the assumption that Design Thinking nurtures team working skills and it revealed that before the workshop, the participants reflected low confidence levels as only $8 \%$ of the participants indicated high levels of confidence. This changed however after the participants finished the DTGW as evidenced by the fact that $28 \%$ of the participants reported feeling completely confident.

\subsection{Creativity Facilitation in Others}

A closely related competence is the openness to help others be more creative. As put forth by the CBCA, the participants are expected to facilitate and/or help others foster their own creative 
processes. In this regard, the study reflects a substantial improvement in the post-survey results as over $60 \%$ (40\% Digital Engineering students) reported being very confident or completely confident in their ability to help others be creative.

\subsection{Testing and Prototyping}

Design Thinking is based on a circular process of prototyping, testing, iterating, and refining a product or process. In order to do this, one must be able to try an approach to a problem or task that they know may not be the final or best solution. The key is to keep testing and not being too attached to the prototype. Addressing this issue, the study looked at the participants' confidence in their ability to try different approaches even if they do not lead to the ultimate solution. Findings from both workshops show that the participants were initially skeptical about their ability to iterate solutions as more than $50 \%$ of them marked themselves in the categories of a little confident and moderately confident. However, the post-survey indicated that $12 \%$ of the participants felt completely confident in their abilities. Nevertheless, the data also shows that more participants felt less confident after the workshop than they did before. During our observation, we noticed that some participants did not get along with one or more teammates. Therefore, this result could be based on personal reflections or a negative experience at the workshop.

\subsection{Perseverance after Failure}

A related trait is that people practicing Design Thinking should not fear failure. On the contrary, failure is a part and a parcel of the process. Nevertheless, prior research showed that perseverance after failure is a trait that's acquired overtime and depends on the past experiences (Henry, 2019; Lent, 2002; Heine, 2001; Hjeltnes, 2015 \& Harackiewicz, 2000). Analyzing the impact of Design Thinking on cultivating the perspective of failure as an opportunity, the study found that engaging with Design Thinking does not have a significant influence on reducing the fear of failure. The post-survey shows a marginal improvement as only $24 \%$ of the participants reported feeling completely confident. Whereas, the rest remained the same. This concurs with previous research indicating the need for more time in order to foster resilience against failure.

\subsection{Mastery of the Creative Process}

Taking responsibility for one's own creative process is a highly sought-after competence. The Design Thinking process as delivered through the D-School teaching model is thought to have been successful at nurturing this in students. However, little evidence has been garnered to this end. Yet, this study shows some promising results with regards to the potential impact of Design Thinking as a method of experiential education. The results highlight that $16 \%$ of the participants lacked the confidence in their ability to take responsibility of their own creativity. Whereas this changed in the post-survey as no participants reported feeling less confident. The positive impact is also reflected in $64 \%$ of the respondents indicating that they felt very confident after the workshop as opposed to only $36 \%$ before the workshop, the majority comes from Digital Engineering students' respondents.

\subsection{Describing the Creative Process}

Another inwardly reflective competence is the ability to explicitly define or describe one's own creative process. In applying the Design Thinking process, participants are expected to get 
better acquainted with themselves and eventually find out what works for them and what does not. The survey supported this assumption by showing that before participating in the GDTW, $28 \%$ of the participants were skeptical about the knowledge of their own creative process. However, this shifted in the post-survey whereby no one expressed such doubts. On the contrary, the post-survey showed $16 \%$ of the participants feeling completely confident as opposed to $0 \%$ in the pre-survey.

\subsection{Successful Creative Problem-Solving}

The main assumption behind this study is that the Design Thinking process cultivates creative problem-solving. The study implies that experiencing the Design Thinking process can engender a boost in confidence in the participant's ability to generate solutions that others would find creative. The pre-test showed that the bulk of the participants $(63 \%)$ rated themselves on the lower end of the confidence scale, whereas the post-test shows the shift in confidence levels with most of the participants $(60 \%)$ indicating feelings of being completely confident and very confident. Our observation of the teams over the one-week event duration confirms that.

\subsection{Openness to working in multidisciplinary teams}

Previous research has emphasized that the diversity of team participants' knowledge, behavior, and values can promote creativity (Hofhuis, 2016; Craig \& Kelly, 1999; Kurtzberg, 2005; Milliken \& Martins, 1996). This study steps back and attempts to analyze the influence of Design Thinking on increasing students' openness to work in multidisciplinary teams. Using the CBCA questionnaire logic but instead of confidence, those set of questions examine the extent to which the participants are comfortable, the study points to an increase in openness to working in multidisciplinary teams following the exposure to the Design Thinking approach, especially among Digital Engineering students. When asked about the extent to which the participants are comfortable in working with teams from other educational backgrounds, initially the participants seemed doubtful as over $50 \%$ of them said that they did not mind. This perspective changed after they engaged with multidisciplinary teams at the GDTW in 2017, as evidenced by around $70 \%$ of the participants expressing full comfort in working with people from other educational backgrounds. It is worth mentioning that 2 participants indicated feeling a little comfortable after the workshop as opposed to only one participant saying so in the presurvey. This could be explained by unfavorable group dynamics or simply a negative experience that led to a bad impression. However, the overall result of both workshops (2017 and 2018) indicates a relative improvement.

A closely related question is addressing the openness to work with people from other professions. The survey results also showed improvement especially in a clear shift in perspective from $52 \%$ of the participants not minding to $64 \%$ feeling fully comfortable. Similar results are reported on the question about working with diverse age groups, whereby participants initially did not mind and then later they felt more comfortable.

\subsection{Openness to working with culturally diverse teams}

The question of cultural diversity is a more sensitive one and research in this area is often subject to social desirability bias (Krumpal, 2013). Many do not fully admit their true feelings to avoid judgement or accusation (Sogancilar, 2018). In the case of this study, the anonymity 
was ensured to assure honest responses. In doing so, the study found evidence to support the initial hypothesis indicating that the engagement with the Design Thinking process helps people emphasize with each other. The causality cannot be established using the data collected here but what can be reported is that people felt more comfortable in working with people from other cultural backgrounds after having been exposed to the Design Thinking teaching modality.

\section{Conclusions and Future Work}

This study brings us a step closer towards gaining a better understanding of the impact of the Design Thinking process on cultivating creative confidence among students in general and Digital Engineering students in particular. By focusing on the problem-solving approach at the core of Design Thinking, the study showed that the creative agency of both the Digital Engineering students, and also the other participants of the GDTW can be marginally enhanced by means of experiential learning and engaging with Design Thinking processes in multidisciplinary teams. Moreover, the research suggests that engaging students in multicultural teams can help improve their propensity to work with people who come from different backgrounds. This study also showed that the method put forth by Royalty et al. (2014) to evaluate the creative agency proved useful in gauging insights on the participants' self-reported creative self-efficacy and perception of their own creative capacity.

Future work includes replications of the CBCA assessment tool in other GDTW programs, which are needed to consolidate the results generated here. These will also be enriched with interviews to gather more in-depth information about the reasoning behind the participants' responses. Moreover, future research will attempt to generate both qualitative and quantitative evidence for the effects of the D-School methodology on the participants' creative agency and career outcomes. This is essential to pave the way for an evidence-based expansion of the method both at the D-School and at other organizations throughout the world.

\section{References}

Buchanan, R. (1992). Wicked problems in design thinking. Design issues, vol.8, no.2, pp. 521.

Craig, T. Y. \& Kelly, J. R. (1999). Group cohesiveness and creative performance. Group Dynamics: Theory, Research, and Practice, vol. 3, pp. 243-256.

Cross, N. (2007). Designerly ways of knowing. Board of international research in design. Basel: Birkhiuser, pp. 41.

Dunne, D., \& Martin, R. (2006). Design thinking and how it will change management education: An interview and discussion. Academy of Management Learning \& Education, vol. 5, no. 4, pp. 512-523

Harackiewicz J. M., Barron K. E., Tauer J. M., Carter S. M., Elliot A. J. (2000). Short-term and long-term consequences of achievement goals: Predicting interest and performance over time. Journal of Educational Psychology, vol. 2, pp. 316-330.

Henry, M. A., Shorter, S., Charkoudian, L., Heemstra, J. M., \& Corwin, L. A. (2019). FAIL Is Not a Four- Letter Word: A Theoretical Framework for Exploring Undergraduate Students' Approaches to Academic Challenge and Responses to Failure in STEM Learning Environments. CBE life sciences education, vol. 18, no.1.

Heine S. J., Kitayama S., Lehman D. R., Takata T., Ide E., Leung C., Matsumoto H. (2001). Divergent consequences of success and failure in Japan and North America: 
An investigation of self-improving motivations and malleable selves. Journal of Personality and Social Psychology, vol. 4, pp. 599-615.

Hjeltnes A., Binder P. E., Moltu C., Dundas I. (2015). Facing the fear of failure: An explorative qualitative study of client experiences in a mindfulness-based stress reduction program for university students with academic evaluation anxiety. International Journal of Qualitative Studies on Health and Well-Being, vol. 1, pp. 27990.

Hofhuis, J., van der Rijt, P. G., \& Vlug, M. (2016). Diversity climate enhances work outcomes through trust and openness in workgroup communication. SpringerPlus, vol. 5, no. 1, pp. 714.

Kelley, T., \& Kelley, D. (2013). Creative confidence: unleashing the creative potential within us all. London: W. Collins. pp. 2.

Krumpal, I. Determinants of social desirability bias in sensitive surveys: a literature review (2013). Qual Quant, vol. 47, pp. 2025-2047.

Kurtzberg, T. R. (2005). Feeling creative, being creative: An empirical study of diversity and creativity in teams. Creativity Research Journal, vol. 17, pp. 51-65.

Lent R. W., Brown S. D., Hackett G. (2002). Social cognitive career theory. Career Choice and Development, pp.255-311.

Milliken, F. J. \& Martins, L. L. (1996). Searching for common threads: Understanding the multiple effects of diversity in organizational groups. Academy of Management Review, no.21, pp. 402-433.

Royalty, A., Noelle Oishi, L., \& Roth, B. (2014). Acting with Creative Confidence: Developing a Creative Agency Assessment Tool. In C. Meinel \& L. Leifer (Eds.), Design Thinking Research: Building Innovation Eco-Systems, pp.85-101. Springer.

Sogancilar, N., Ors, H. (2018). Understanding the challenges of multicultural team management. Journal of Business, Economics and Finance (JBEF), vol.7, no.3, pp. 259-268.

Traifeh, H., Staubitz, T., \& Meinel, C. (2019, October). Improving learner experience and participation in MOOCs: A design thinking approach. In 2019 IEEE Learning With MOOCS (LWMOOCS). IEEE. pp. 165-169 